\title{
Major depressive disorder is characterized by greater reward network activation to monetary than pleasant image rewards
}

\author{
Moria J. Smoski ${ }^{A},{ }^{*}$, Alison Rittenberg ${ }^{B}$, and Gabriel S. Dichter ${ }^{A, B, C, D}$ \\ A Department of Psychiatry and Behavioral Sciences, Duke University Medical Center, Durham \\ NC 27710 \\ B Carolina Institute for Developmental Disabilities, University of North Carolina at Chapel Hill \\ School of Medicine, Chapel Hill, NC 27599 \\ C Department of Psychiatry, University of North Carolina at Chapel Hill School of Medicine, \\ Chapel Hill, NC 27599 \\ D Duke-UNC Brain Imaging and Analysis Center, Duke University Medical Center, Durham, NC, \\ 27710
}

\begin{abstract}
Anhedonia, the loss of interest or pleasure in normally rewarding activities, is a hallmark feature of unipolar Major Depressive Disorder (MDD). A growing body of literature has identified frontostriatal dysfunction during reward anticipation and outcomes in MDD. However, no study to date has directly compared responses to different types of rewards such as pleasant images and monetary rewards in MDD. To investigate the neural responses to monetary and pleasant image rewards in MDD, a modified Monetary Incentive Delay task was used during fMRI scanning to assess neural responses during anticipation and receipt of monetary and pleasant image rewards. Participants included nine adults with MDD and thirteen affectively healthy controls. The MDD group showed lower activation than controls when anticipating monetary rewards in right orbitofrontal cortex and subcallosal cortex, and when anticipating pleasant image rewards in paracingulate and supplementary motor cortex. The MDD group had relatively greater activation in right putamen when anticipating monetary versus pleasant image rewards, relative to the control group. Results suggest reduced reward network activation in MDD when anticipating rewards, as well as relatively greater hypoactivation to pleasant image than monetary rewards.
\end{abstract}

\section{Keywords}

Major Depressive Disorder; Reward; Anhedonia; Anticipation; Magnetic Resonance Imaging

\section{Introduction}

Anhedonia, the loss of interest or pleasure in normally rewarding activities, is a hallmark feature of unipolar Major Depressive Disorder (MDD; American Psychiatric Association,

(C) 2011 Elsevier Ireland Ltd. All rights reserved.

*Address correspondence by moria.smoski@duke.edu, or by mail to: Moria Smoski, Duke University Medical Center, DUMC Box 3026, Durham NC 27710. Phone: 919.684.6717; Fax: 919.684.6770.

Publisher's Disclaimer: This is a PDF file of an unedited manuscript that has been accepted for publication. As a service to our customers we are providing this early version of the manuscript. The manuscript will undergo copyediting, typesetting, and review of the resulting proof before it is published in its final citable form. Please note that during the production process errors may be discovered which could affect the content, and all legal disclaimers that apply to the journal pertain. 
1994). A growing body of literature has identified frontostriatal dysfunction during reward anticipation and reward outcomes in MDD. To date, five studies of reward processing in MDD have investigated responses during different temporal phases of reward processing. Forbes and colleagues (2006) reported that children with MDD demonstrated decreased orbitofrontal cortex, anterior cingulate, amygdala, and caudate activation during both reward anticipation and feedback, but found little evidence of differential effects contingent on the temporal phase of the response. Forbes and colleagues (2009) reported reduced striatal activation in depressed adolescents during reward anticipation and reward outcome that predicted positive affect in natural environments. Knutson and colleagues (2008) employed a monetary incentive delay task and found no striatal activation differences between adult groups during reward anticipation, but increased anterior cingulate activation during anticipation of monetary gains in the MDD group. Again using a monetary incentive delay task in adults with or without MDD, Pizzagalli and colleagues (2009) found relatively reduced putamen activation during reward anticipation and reduced activation in nucleus accumbens and caudate during receipt of reward.

Finally, our own research group reported anomalous neural responses during reward selection, anticipation, and feedback in adults with MDD (Smoski et al., 2009) using a Wheel-of-Fortune task (Ernst et al., 2004). The MDD group was characterized by reduced activation of striatal regions during reward selection, reward anticipation, and reward feedback, by hyperresponsivity in orbitofrontal cortex during reward selection, and by decreased activation of the middle frontal gyrus and the anterior cingulate cortex during reward selection and anticipation. This study demonstrated unique regions of functional deficits in MDD during different temporal phases of reward processing, and, most critically, that striatal dysfunction in MDD was evident during reward anticipation.

Functional neuroimaging studies of responses to the presentation of pleasant stimuli not contingent on participant behavior in MDD indicate anomalous responses in the striatum (Keedwell et al., 2005b; Mitterschiffthaler et al., 2003; Schaefer et al., 2006) as well as the medial prefrontal cortex (Keedwell et al., 2005a; Kumari et al., 2003; Schaefer et al., 2006), and the pregenual and subgenual anterior cingulate (Keedwell et al., 2005b; Kumari et al., 2003). Notably, a study of participants who were recovered from MDD, and therefore no longer impacted by mood state or current medication usage, also revealed reduced activation to pleasant taste and picture stimuli in reward areas such as ventral striatum compared to healthy controls (McCabe et al., 2009). However, none of these studies directly compared responses to monetary rewards and other pleasant stimuli, nor were differential responses during anticipation and outcomes assessed.

As the field moves from investigations of MDD-related reward network abnormalities to a more fine-grained analysis of their nature, function, and clinical implications, it is important to understand the relative impact of different reward types on brain activation patterns. The studies of reactivity to pleasant stimuli reviewed above focused on putatively pleasurable stimuli (e.g., images, words, and/or memories; Keedwell et al., 2005a; Keedwell et al., 2003; Kumari et al., 2003; Mitterschiffthaler et al., 2003; Schaefer et al., 2006), while studies of reward processes have exclusively relied upon monetary rewards (Dichter et al., 2009; Forbes et al., 2006; Forbes et al., 2009; Knutson et al., 2008; Pizzagalli et al., 2009; Smoski et al., 2009). Recent work by Kessler and colleagues (2011) emphasized the importance of the personal relevance of stimuli in understanding depression-specific activation in limbicparalimbic regions. Minimal work to date has addressed the differential impact of reward types, despite the potential clinical implications of such differences. For example, cognitive and behavioral treatments for MDD emphasize engagement with activities that promote pleasurable (associated with a sense of enjoyment) and mastery (associated with a sense of accomplishment) experiences (e.g., Beck et al., 1979; Jacobson et al., 1996). Theoretically, 
supported engagement with these activities is believed to become self-reinforcing and to form new behavioral patterns that can alleviate anhedonic symptoms (Martell et al., 2001). However, there is a dearth of information about what experiences are most likely to be effective in forging these behavioral patterns, a process that may be mediated by relative reward network responsivity to stimulus types.

The goal of the present study was to compare reward network activation in MDD during anticipation and receipt of monetary and pleasant image rewards (derived from the International Affective Pictures Set). Based on previous studies of reward processes in MDD (Knutson et al., 2008; Smoski et al., 2009), we hypothesized that the MDD group would be characterized by striatal and anterior cingulate hypoactivation while anticipating monetary reward and medial prefrontal hypoactivation to monetary reward outcomes. Hypotheses about responses to pleasant stimuli were shaped by findings that MDD is characterized by medial prefrontal and striatal hypoactivation while viewing pleasant images (Mitterschiffthaler et al., 2003; Schaefer et al., 2006). Though no previous studies have directly compared monetary to pleasant image rewards MDD, we hypothesized that in the context of a reduced capacity to experience pleasure, a pleasant image would be less salient than a reward with concrete external/tangible value (such as money). Thus, we hypothesized that monetary rewards would be associated with relatively greater reward network activation than pleasant image rewards in the MDD group.

\section{Method}

\subsection{Participants}

Thirteen affectively healthy adult control participants ( $26.2 \pm 6.3$ years old; all right-handed) were recruited from lists of potential participants maintained by the Duke-UNC Brain Imaging and Analysis Center (BIAC). Nine adults with MDD (34.4 \pm 15.0 years old; 8 right-handed) were recruited via the Cognitive Behavioral Research and Treatment Program at Duke University Medical Center and via online advertisements. Within the MDD group, four participants were taking antidepressant medication (SSRIs or SNRIs). One MDD participant had a prior hospitalization. One MDD participant was in the first depressive episode, three were in their second, third, or fourth episode, and the remainder reported 5 or more episodes. One MDD participant met criteria for a comorbid generalized anxiety disorder, and another for comorbid binge eating disorder.

Exclusion criteria for both groups included age $<18$ or $>55$ years, current or past psychosis or mania, estimated verbal IQ scores $<80$, or MRI contraindications (e.g., presence of metal in body) as assessed by an MRI safety questionnaire. Groups did not differ in age, racial/ ethnic background, or IQ, $p$ 's $>0.05$ (see Table 1). Inclusion in the MDD group was contingent on a diagnosis of current MDD based on SCID I semi-structured interview (First et al., 1996). Control participants were lifetime-free of MDD and currently free of all Axis I disorders based on SCID I interview. All participants consented to a protocol approved by the local Human Investigations Committees at both UNC-Chapel Hill and Duke University Medical Centers, and participants were paid at least $\$ 35$ for completing the imaging portion of the study. All participants had normal or corrected-to-normal vision and completed a mock scan session.

\subsection{FMRI task}

The fMRI task was a modified from the Monetary Incentive Delay (MID) task as implemented in Knutson, Westdorp, Kaiser, \& Hommer (2000). Participants practiced the task outside the scanner prior to imaging. Participants completed four functional imaging runs. Two runs were the standard implementation of the "win version" of the MID (i.e., 
money could be won or not won, but money could not be lost). Two runs were modified so that participants could "win" the opportunity to view a pleasant image instead of a neutral image. Run types (i.e., "money" runs and "image" runs) were presented in alternating order, and the run type presented first was counter-balanced across participants. Each run began with a 10-sec instructional screen indicating whether the forthcoming run would be a "money" or an "image" run. The two reward types (i.e., money and images) were segregated by run to minimize the number of cues to be memorized (i.e., separating by run type required learning only two cues (i.e., potential win trial, or not), whereas intermixing within runs would require four cues (i.e., money win, image win, money non-win, image nonwin) $)^{1}$.

Task conditions and trial timings are summarized in Figure 1. Each trial consisted of: (1) a $2000 \mathrm{~ms}$ cue that indicated whether adequately quick responses to the forthcoming target bulls-eye could result in a "win" or "non-win" (a triangle) or could not (a circle); (2) a delay period during which a crosshair was presented for 2000-2500 msec; (3) a target bulls-eye that required a speeded button press presented for up to $500 \mathrm{msec}$; (4) $3000 \mathrm{~ms}$ of feedback that indicated whether that trial was a "win" or not; and (5) a variable length ITI crosshair presented such that the total duration of each trial was $12 \mathrm{sec}$. Trial types (i.e., potential win or no potential win) were aperiodic and pseudorandomly ordered. Each 8-minute run contained 40 trials: 20 were potential win trials, 20 were non-win control trials.

During money runs, participants could win $\$ 1$ per trial, and feedback was a text display of the amount of money won (e.g., "+\$1”). During image runs, participants viewed a single, high-resolution pleasant or neutral image. Coincident with this feedback, a cumulative count of the number of dollars won or number of pleasant images viewed within the run was presented. Participants were instructed to respond to all target bullseyes as quickly as possible, and outcomes were contingent on reaction times. The task was adaptive such that participants were successful on two-thirds of trials, regardless of individual differences in reaction times.

Prior to entering the scanner, participants were shown the money they could win based on scanner task performance and rated images on the dimensions of Valence and Arousal using the Self Assessment Manikin (Bradley and Lang, 1994), a 9-point Likert non-verbal pictorial assessment technique. This untimed assessment allowed participants to become familiar with the potential rewards to be presented during the imaging task. Participants were informed that they would receive the total amount of money won. All stimuli were presented using E-Prime presentation software v. 1.1 (Psychology Software Tools Inc., Pittsburgh, PA) and displayed in the scanner through magnet-compatible goggles (Resonance Technology, Inc., Northridge CA).

\subsection{Image stimuli}

Two image sets (pleasant and neutral) were derived from images within the International Affective Picture Set (Lang et al., 2008). The pleasant set was comprised of images with Lang mixed-gender valence ratings between 6.1 and 9.0, with mean (SD) normative valence ratings $=7.24(0.57)$ and arousal ratings $=4.23(0.59)$ on a $1-9$ scale. Images included a mix of social scenes, infants/children, and nature images. Given that valence ratings varied by sex for erotica in published norms, erotic images were not included. The pleasant set included 51 images. The neutral set was comprised of images with Lang mixed-gender

\footnotetext{
${ }^{1}$ It should be noted that though the use of the same cue for both reward types has the advantage of minimizing memory burden or confusion on the part of participants, it confounds any conditioning-based learning between reward types. We thank an anonymous reviewer for raising this point.
} 
valence ratings between 4.5 and 6.0. This set included 46 images (normative mixed-gender mean valence ratings $=5.08(0.58)$ and arousal ratings $=3.38(0.60))$.

\subsection{Imaging methods}

Scanning was performed on a General Electric 4T LX NVi MRI scanner system equipped with $41 \mathrm{mT} / \mathrm{m}$ gradients (General Electric, Waukesha, Wisconsin, USA). A quadrature birdcage radio frequency head coil was used for transmit and receive. A high resolution T1weighted image with 68 slices was acquired using a 3D fast SPGR pulse sequence $(\mathrm{TR}=$ $500 \mathrm{~ms} ; \mathrm{TE}=20 \mathrm{~ms} ; \mathrm{FOV}=24 \mathrm{~cm}$; image matrix $=256 \times 256$; voxel size $=1.67 \mathrm{~mm}^{3}$ ) and used for coregistration with the functional data. This structural image was aligned in a near axial plane defined by the anterior and posterior commissures. Whole brain functional images were acquired using an Echo Planar Imaging pulse sequence sensitive to blood oxygenation level dependent contrast (TR, $1500 \mathrm{~ms}$; TE, $31 \mathrm{~ms}$; FOV, $24 \mathrm{~cm}$; image matrix, $64 \times 64 ; \alpha=62^{\circ}$; voxel size $=53.4375 \mathrm{~mm}^{3} ; 34$ axial slices). Functional images were aligned similarly to the T1-weighted structural image. A semi-automated high-order shimming program ensured global field homogeneity.

\subsection{Imaging data analysis}

Functional data were preprocessed using FSL version 4.0.4 (Oxford Centre for Functional Magnetic Resonance Imaging of the Brain (FMRIB), Oxford University, U.K.). Timing files were converted to FSL compatible format and NIFTI image data files were generated. Preprocessing was applied in the following steps: (i) brain extraction for non-brain removal (Smith et al., 2004), (ii) motion correction using MCFLIRT (Smith, 2002), (iii) spatial smoothing using a Gaussian kernel of FWHM 5 mm, (iv) mean-based intensity normalization of all volumes by the same factor, and (v) high-pass filtering (Jenkinson et al., 2002). Functional images of each participant were co-registered to structural images in native space, and structural images were normalized into a standard stereotaxic space (Montreal Neurological Institute) for intersubject comparison. The same transformation matrices used for structural-to-standard transformations were then used for functional-tostandard space transformations of co-registered functional images. All registrations were carried out using an intermodal registration tool (Jenkinson et al., 2002; Smith et al., 2004). Voxel-wise temporal autocorrelation was estimated and corrected using FMRIB's Improved Linear Model (FILM; Jenkinson and Smith, 2001).

Onset times of events were used to model a signal response containing a regressor for each response type, which was convolved with a double- $\gamma$ function to model the hemodynamic response. Model fitting generated whole brain images of parameter estimates and variances, representing average signal change from baseline (activation; positive regressor) and below baseline (deactivation; negative regressor). Group-wise activation and deactivation images were calculated by a mixed effects higher level analysis using Bayesian estimation techniques, FMRIB Local Analysis of Mixed Effects (FILM, Smith et al., 2004; Woolrich et al., 2001). Following the guidelines of Lieberman and Cunningham (2009), clusters of ten or more voxels with minimum values of $z>2.58(p<0.005)$ were identified using customized MATLAB scripts.

\section{Results}

\subsection{Image ratings}

A 2 (Image Type: pleasant, neutral) $\times 2$ (Group: MDD, control) MANOVA was performed for both valence and arousal ratings. For valence ratings, there was a significant main effect of image type, $F(1,19)=70.79, p<.00001$, with pleasant images rated as more positive than neutral images. There was no significant main effect of group, $F(1,19)=0.77, p=0.64$, and 
the Image Type $\times$ Group interaction was not significant, $F(1,19)=0.17, p=0.68$. For arousal ratings, no significant main effects or interactions were observed, $p$ 's $>0.20$.

\subsection{Reaction time}

A 2 (Reward Type: image, money) $\times 2$ (Group: MDD, control) mixed-model MANOVA was performed to model button press reaction times in the scanner. There was a main effect of reward type, $F(1,20)=12.53, p=0.002$, such that reaction times were significantly faster to money than image trials. There was no significant main effect of group or group $\times$ reward type interaction, $F(1,20)=0.20, p=0.66$ and $F(1,20)=0.33, p=0.57$, respectively. A second set of MANOVAs was performed for each reward type, comparing trials for which success was or was not possible (i.e., control trials). For both image and money trials, there was a significant main effect of success possibility, $F(1,20)=31.40, p=0.00002$ and $F(1$, $20)=.22 .75, p=0.0001$, respectively, such that participants responded more quickly when cued that success was possible. There were no significant main effects of group or success $x$ group interactions, $\mathrm{p}$ 's $>0.60$.

\subsection{Imaging data}

Anticipation and outcome phases were analyzed separately using a whole-brain analytic approach. Within- and between-group analyses were performed for both monetary and pleasant image rewards, examining potential win versus non-potential win contrasts during the anticipatory phase and for wins versus non-win contrasts during the outcome phase. In addition, Group $\times$ Reward type interaction analyses were performed. Between-groups and interaction results were masked by whole-sample (i.e., both groups combined) activation maps of Reward (images + money, images, or money) > Non-Reward contrasts for the appropriate task phase, therefore limiting significant results to regions activated by the task. See Supplemental Figure 1 for regions included in these masks. Within-group analyses were conducted to determine if the task elicited activation in typical reward-related regions for both MDD and control groups, consistent with previous literature (e.g., Knutson et al., 2008). A more conservative $z$ score threshold of 3.5 was used for within-subjects analyses. Tables of within-groups results for both monetary and pleasant image rewards are available from the authors upon request. Activation localizations were based on Harvard-Oxford cortical and subcortical structural probabilistic atlases, with Brodmann area identification via Talairach Daemon, as implemented in FSLView v3.0.

\subsection{Within-Groups Results for Monetary Rewards}

With regards to anticipation of monetary win versus non-win trials in the control group, results were largely consistent with previous studies (e.g., Knutson et al., 2008). Activation was observed in bilateral thalamus, extending to brainstem and through the striatum including caudate and nucleus accumbens to the subcallosal cortex. Activation was also observed in dorsal anterior cingulate and paracingulate gyrus, extending to supplementary motor cortex, as well as in left orbitofrontal cortex. In addition to the thalamo-striatal and frontal activations, clusters were observed in bilateral cerebellum, occipital fusiform, lateral occipital cortex, supramarginal gyrus, insula, and right precuneus and superior parietal lobule. MDD participants showed activation in bilateral thalamus, pallidum, caudate, accumbens and putamen; bilateral insula, with activation extending to orbitofrontal cortex on the left; anterior cingulate; and supplementary motor cortex; as well as bilateral cerebellum, right central opercular cortex, and left lateral occipital cortex.

With regards to monetary win versus non-win outcomes, win outcomes were associated with activation in right insula and lingual gyrus in both control and MDD groups. In addition, left cerebellum and occipital fusiform were activated in the control group. 


\subsection{Within-Groups Results for Pleasant Image Rewards}

With regards to anticipation of pleasant image win versus non-win trials in the control group, anticipation of a positive image was associated with activation in right caudate and bilateral supplementary motor cortex. Winning outcomes were associated with activation in right intercalcarine cortex. Pleasant image win versus non-win contrasts were not associated with activation in any regions in the MDD group for either anticipation or outcome phases.

\subsection{Within-Groups Comparisons between Reward Types}

Within-group analyses were conducted to compare activations between reward types. Activation tables are available from the authors by request; see Supplemental Figure 2. Within the control group, regions that showed greater activation to anticipation of monetary than pleasant image rewards included right subcallosal cortex, bilateral anterior paracingulate gyrus, left anterior cingulate, bilateral insula, bilateral hippocampus, left central opercular cortex, parahippocampal gyrus extending to lingual gyrus, bilateral thalamus extending to caudate, right precuneus, several bilateral regions of cerebellum, and regions of occipital cortex. Among the MDD group, greater activation to monetary than pleasant image rewards was observed in bilateral anterior cingulate and right paracingulate, right insula, bilateral parahippocampal gyrus, left thalamus, right putamen, left pallidum, right supramarginal gyrus, and several bilateral regions of cerebellum and occipital cortex. Neither the control group nor the MDD group demonstrated regions of greater activation to pleasant image than monetary rewards.

In the outcome phase, the control group showed greater activation to pleasant image than monetary rewards in right precentral gyrus, superior parietal lobe, and lateral occipital cortex. No regions showed greater activation to monetary rewards. In the MDD group, greater activation was observed for monetary than pleasant image rewards in right precuneus, right lateral occipital cortex, bilateral lingual gyrus, and right cerebellum. No regions showed greater activation to pleasant image than monetary rewards in the MDD group.

\subsection{Between-Groups Comparisons for Monetary Rewards}

Comparisons between the MDD and control groups were performed separately for anticipation of win versus non-win monetary rewards and for actual win versus non-win monetary outcomes. For anticipation, MDD participants showed greater activation than controls in a medial region of frontal pole. Control participants showed greater activation than the MDD group in anterior cingulate, right orbitofrontal cortex, right lateral frontal pole, left frontal operculum, left hippocampus, left planum polare, bilateral temporal pole, and regions of occipital cortex. See Table $2 \mathrm{~b}$. For outcome, no regions showed greater activation for MDD than control participants. Control participants showed greater activation than the MDD group in right temporal pole (see Table $3 b$ ).

\subsection{Between-Groups Comparisons for Pleasant Image Rewards}

Comparisons between the MDD and control groups were performed separately for anticipation of win versus non-win pleasant image rewards and for win versus non-win pleasant image outcomes. For both anticipation and outcome, no regions showed greater activation for MDD than control participants. For anticipation, control participants showed greater activation than the MDD group in clusters in the anterior cingulate cortex and paracingulate gyrus, right middle frontal gyrus, right pallidum, right parahippocampal gyrus, bilateral precentral gyrus, precuneus, and areas of brainstem (see Table 2c). For outcome, control participants showed greater activation than the MDD group in clusters in bilateral frontal pole, anterior cingulate cortex, right caudate and putamen, right precentral gyrus, 
bilateral precuneous, right posterior cingulate gyrus, right intracalcarine cortex, left lateral occipital cortex, and bilateral occipital fusiform gyrus (see Table $3 \mathrm{c}$ ).

\subsection{Anticipatory Responses to Monetary versus Pleasant Image Rewards}

One striatal region, the right putamen, showed a significant Group $\times$ Reward Type interaction, with MDD participants showing a stronger Money > Pleasant Image contrast than controls during win anticipation. Regions that showed greater Money > Pleasant Image activation for control than MDD participants included clusters within right hippocampus, thalamus, and occipital pole (see Figure 2 and Table 2a).

\subsection{Monetary versus Pleasant Image Reward Outcomes}

Regions that showed a significant Group $\times$ Reward Type interaction, with MDD participants showing a stronger Money > Pleasant Image contrast than controls during win versus nonwin outcomes, included clusters within the right precentral gyrus, insula, intracalcarine cortex, several occipital regions, and right cerebellum. No regions showed greater Money > Pleasant Image contrasts for control than MDD participants (see Figure 2 and Table $3 a)^{2}$.

\section{Discussion}

The goal of this study was to compare neural responses to monetary and pleasant image rewards in individuals with and without MDD. Differences in reward network activation were observed between MDD and control participants during reward anticipation for both monetary and pleasant image rewards. Specifically, the MDD group showed hypoactivation in right orbitofrontal cortex and subcallosal cortex during anticipation of monetary rewards. For anticipation of pleasant image rewards, the MDD group showed hypoactivation in anterior paracingulate gyrus and supplementary motor cortex, which may be associated with preparing for task-related action. Previous studies have noted hypoactivation in MDD versus control participants during monetary reward anticipation in right orbitofrontal cortex (Forbes et al., 2006), anterior cingulate, and subcallosal cortex (Smoski et al., 2009), though hyperactivation in anterior cingulate has also been observed (Knutson et al., 2008). These findings support the hypothesis that disruptions in fronto-cingulate-striatal networks during reward anticipation are associated with MDD.

No differences were observed between MDD and control participants in reward network activation during the outcome phase. These findings are counter to previous studies which have observed decreased fronto-cingulate-striatal activation during receipt of reward in MDD participants (Forbes et al., 2006; Knutson et al., 2008; Pizzagalli et al., 2009; Smoski et al., 2009). However, given the small sample size in the current study, as discussed below, null findings should be interpreted with caution.

When responses to monetary and pleasant image rewards were directly compared, monetary rewards elicited greater reward network activation than pleasant image rewards during anticipation in both groups. Specifically, during reward anticipation, the MDD group showed relatively greater activation to monetary than pleasant image rewards in right putamen, compared to controls. Activation in putamen has been associated with stimulusaction-dependent reward prediction (Haruno and Kawato, 2006), and has been found to be reduced in reward anticipation in individuals with MDD (Pizzagalli et al., 2009). These

\footnotetext{
${ }^{2}$ To examine the potential impact of medication usage among the MDD group, beta weight scores were extracted for each subject for reward network regions that showed a significant Money > Image, MDD > Control contrast. For the anticipation phase this included right putamen and bilateral precentral gyrus; for the outcome phase this included two areas of right medial PFC/frontal pole. Beta weight scores were used in a between-groups $t$ test comparing medicated $(n=4)$ with unmedicated $(n=5)$ MDD participants. Medicated and unmedicated participants did not differ in ROI activation, and did not differ in BDI score.
} 
differences suggest that sensitivity to anticipation of monetary rewards may be more preserved than sensitivity to pleasant images in MDD.

Why might monetary rewards elicit a stronger reward-network neural response than pleasant image rewards in MDD? Rewards that are less salient, either by nature of being noncontingent on participant behavior or by reduced intensity, are known to be associated with reduced striatal activation (Zink et al., 2006). In considering the differences between monetary and image rewards, it is interesting to note that the reward value of pleasant images is immediate (i.e., the provocation of a positive emotional experience) whereas receipt of the monetary reward involves both an immediate mood induction as well as response to receipt of a longer-term benefit of receiving money at the end of the experiment. Disturbed cognitions in MDD may include a sense that the individual has not earned or is not worthy of pleasurable experiences (Beck et al., 1979), whereas winning a financial reward may be seen as a step towards ameliorating functional deficits that can accompany MDD (e.g., difficulty working, creating a financial burden by incurring treatment expenses, etc.) This speculative interpretation requires further experimental investigation and replication. However, results suggest that investigations of anhedonia in MDD may find more pronounced differences from control groups when using pleasure-based rather than monetary rewards. In addition, our results serve as a reminder that psychosocial treatments such as behavioral activation that promote engagement with potentially rewarding environments may wish to pay special attention to environments associated with tangible outcomes.

Interpretation of results is limited by the heterogenous nature of the MDD sample, especially by comorbid conditions in two participants, handedness, number of previous depressive episodes, and the use of antidepressant medications by a little less than half of the MDD sample. Given that antidepressant treatments can change reward processing of pleasant taste and images in healthy controls (McCabe et al., 2010), it will be critical to test unmedicated participants in future studies. It is unclear if or how these factors affect our results, and thus they should be interpreted with caution until they can be replicated in a more homogenous sample. Statistically the most likely consequence of a heterogenous sample is a reduction in power, as greater heterogeneity leads to greater error variance. Thus Type 2 is more of a concern than Type 1 error. We focus our interpretation of results on the presence of findings rather than their absence. In addition, we have no direct self-report comparison of the pleasantness or hedonic value of monetary versus pleasant image rewards. Potential win trials were associated with faster button press reaction times than non-reward trials for both image and money runs, suggesting both may function in a similar way to modify behavior. However, faster reaction times to monetary reward than pleasant image reward suggest that the monetary trials were more rewarding. It would be helpful in future studies to obtain subjective reports of pleasantness between the two types of reward. The salience and reward value of images might also be increased by allowing participants to individually select images that are most positive to them. Ideographic image selection might also lead to use of images that are more arousing as well as positive; pleasant images in the current study were rated as more pleasant but equivalently arousing to neutral images. Additionally, future studies are needed to address the differential impact of punishment (e.g., losing money or being shown a negative image) versus non-reward (e.g., not winning money or being shown a neutral image) in MDD.

Finally, while within-groups results were consistent with previous studies for anticipation of monetary rewards and anticipation of pleasant images for controls, outcome results differed somewhat from previous studies. Knutson and colleagues (2008) found that monetary gain versus non-gain outcomes were associated with activation in mesial prefrontal cortex, posterior cingulate cortex, caudate, and hippocampus. Given our small and heterogeneous 
sample, it may be that activations in these regions did not meet the activation threshold for detection. It is notable that one of the regions that did show activation to winning outcomes in our sample, the insula, showed greater activation in controls than MDD participants during winning outcomes in Knutson et al 2008. The lack of differential activation to pleasant image anticipation or outcomes in MDD participants is consistent with the interpretation that MDD participants did not find the pleasant images in the present study rewarding.

In conclusion, this study suggests that MDD is characterized by a relatively greater decrease in activation during anticipation of purely pleasurable rewards, such as the effects of pleasant images, than to tangible rewards such as money. More broadly, these results suggest that the type of stimulus used to elicit reward network activation appears to be an important consideration in studies of MDD. Future behavioral and neuroimaging studies of reward reactivity in MDD may be strengthened by giving careful consideration to the type of reward used, and potential implications for psychosocial treatments bear further investigation.

\section{Supplementary Material}

Refer to Web version on PubMed Central for supplementary material.

\section{Acknowledgments}

The authors thank Dr. Brian Knutson for kindly sharing the MID task, Josh Bizzell, Chris Petty, and Todd Harshbarger for assistance with image analysis, MRI technologists Susan Music, Natalie Goutkin, and Luke Poole for assistance with data acquisition, and Jennifer Felder for assistance with several aspects of this research. Assistance for this study was provided by the Neuroimaging Core of the UNC Neurodevelopmental Disorders Research Center (P30 HD03110). This research was supported by grants from the NARSAD Young Investigator Program. Investigator effort was supported by NIMH K23 MH087754 to M. Smoski and NIMH K23 MH081285 to G. Dichter.

\section{References}

American Psychiatric Association. Diagnostic and statistical manual of mental disorders. Washington, DC: 1994. DSM-IV

Beck, AT.; Rush, AJ.; Shaw, BF.; Emery, G. Cognitive Therapy of Depression. Guildford Press; New York: 1979.

Bradley MM, Lang PJ. Measuring emotion: The Self-Assessment Manikin and the Semantic Differential. Journal of Behavior Therapy and Experimental Psychiatry. 1994; 25:49-59. [PubMed: 7962581]

Dichter GS, Felder J, Petty C, Bizzell J, Smoski MJ. The effects of psychotherapy on neural responses to rewards in major depression. Biological Psychiatry. 2009; 66:886-897. [PubMed: 19726030]

Ernst M, Nelson EE, McClure EB, Monk CS, Munson S, Eshel N, Zarahn E, Leibenluft E, Zametkin A, Towbin K, Blair J, Charney D, Pine DS. Choice selection and reward anticipation: an fMRI study. Neuropsychologia. 2004; 42:1585-1597. [PubMed: 15327927]

First, MB.; Spitzer, RL.; Gibbon, M.; Williams, JBW. Structured Clinical Interview for DSM-IV Axis I Disorders (SCID), Clinician Version; Administration Booklet. American Psychiatric Press; Washington, D.C: 1996.

Forbes EE, Christopher May J, Siegle GJ, Ladouceur CD, Ryan ND, Carter CS, Birmaher B, Axelson DA, Dahl RE. Reward-related decision-making in pediatric major depressive disorder: an fMRI study. Journal of Child Psychology and Psychiatry. 2006; 47:1031-1040. [PubMed: 17073982]

Forbes EE, Hariri AR, Martin SL, Silk JS, Moyles DL, Fisher PM, Brown SM, Ryan ND, Birmaher B, Axelson DA, Dahl RE. Altered striatal activation predicting real-world positive affect in adolescent major depressive disorder. American Journal of Psychiatry. 2009; 166:64-73. [PubMed: 19047324] 
Haruno M, Kawato M. Different Neural Correlates of Reward Expectation and Reward Expectation Error in the Putamen and Caudate Nucleus During Stimulus-Action-Reward Association Learning. Journal of Neurophysiology. 2006; 95:948-959. [PubMed: 16192338]

Jacobson NS, Dobson KS, Truax PA, Addis ME, Koerner K, Gollan JK, Gortner E, Prince SE. A component analysis of cognitive-behavioral treatment for depression. Journal of Consulting and Clinical Psychology. 1996; 64:295-304. [PubMed: 8871414]

Jenkinson M, Bannister P, Brady M, Smith S. Improved optimization for the robust and accurate linear registration and motion correction of brain images. Neuroimage. 2002; 17:825-841. [PubMed: 12377157]

Jenkinson M, Smith S. A global optimisation method for robust affine registration of brain images. Medical Image Analysis. 2001; 5:143-156. [PubMed: 11516708]

Keedwell PA, Andrew C, Williams SC, Brammer MJ, Phillips ML. A double dissociation of ventromedial prefrontal cortical responses to sad and happy stimuli in depressed and healthy individuals. Biological Psychiatry. 2005a; 58:495-503. [PubMed: 15993859]

Keedwell PA, Andrew C, Williams SC, Brammer MJ, Phillips ML. The neural correlates of anhedonia in major depressive disorder. Biological Psychiatry. 2005b; 58:843-853. [PubMed: 16043128]

Keedwell PA, Andrew C, Williams SCR, Brammer MJ, Zelaya F, Phillips ML. The neural correlates of depression. Biological Psychiatry. 2003; 53:171S.

Kessler H, Taubner S, Buchheim A, Munte TF, Stasch M, Kachele H, Roth G, Heinecke A, Erhard P, Cierpka M, Wiswede D. Individualized and clinically derived stimuli activate limbic structures in depression: an FMRI study. PLoS One. 2011; 6:e15712. [PubMed: 21283580]

Knutson B, Bhanji JP, Cooney RE, Atlas LY, Gotlib IH. Neural responses to monetary incentives in major depression. Biological Psychiatry. 2008; 63:686-692. [PubMed: 17916330]

Knutson B, Westdorp A, Kaiser E, Hommer D. FMRI visualization of brain activity during a monetary incentive delay task. Neuroimage. 2000; 12:20-27. [PubMed: 10875899]

Kumari V, Mitterschiffthaler MT, Teasdale JD, Malhi GS, Brown RG, Giampietro V, Brammer MJ, Poon L, Simmons A, Williams SC, Checkley SA, Sharma T. Neural abnormalities during cognitive generation of affect in treatment-resistant depression. Biological Psychiatry. 2003; 54:777-791. [PubMed: 14550677]

Lang, PJ.; Bradley, MM.; Cuthbert, BN. Technical Report A-8. Gainesville, FL: University of Florida; 2008. International affective picture system (IAPS): Affective ratings of pictures and instruction manual.

Lieberman MD, Cunningham WA. Type I and Type II error concerns in fMRI research: Re-balancing the scale. SCAN. 2009; 4:423-428. [PubMed: 20035017]

Martell, CR.; Addis, ME.; Jacobson, NS. Depression in Context: Strategies for Guided Action. W.W. Norton; New York, NY: 2001.

McCabe C, Cowen PJ, Harmer CJ. Neural representation of reward in recovered depressed patients. Psychopharmacology (Berl). 2009; 205:667-677. [PubMed: 19529923]

McCabe C, Mishor Z, Cowen PJ, Harmer CJ. Diminished neural processing of aversive and rewarding stimuli during selective serotonin reuptake inhibitor treatment. Biological Psychiatry. 2010; 67:439-445. [PubMed: 20034615]

Mitterschiffthaler MT, Kumari V, Malhi GS, Brown RG, Giampietro VP, Brammer MJ, Suckling J, Poon L, Simmons A, Andrew C, Sharma T. Neural response to pleasant stimuli in anhedonia: an fMRI study. Neuroreport. 2003; 14:177-182. [PubMed: 12598724]

Pizzagalli DA, Holmes AJ, Dillon DG, Goetz EL, Birk JL, Bogdan R, Dougherty DD, Iosifescu DV, Rauch SL, Fava M. Reduced Caudate and Nucleus Accumbens Response to Rewards in Unmedicated Individuals With Major Depressive Disorder. American Journal of Psychiatry. 2009; 166:702-710. [PubMed: 19411368]

Schaefer HS, Putnam KM, Benca RM, Davidson RJ. Event-related functional magnetic resonance imaging measures of neural activity to positive social stimuli in pre- and post-treatment depression. Biological Psychiatry. 2006; 60:974-986. [PubMed: 16780808]

Smith SM. Fast robust automated brain extraction. Human Brain Mapping. 2002; 17:143-155. [PubMed: 12391568] 
Smith SM, Jenkinson M, Woolrich MW, Beckmann CF, Behrens TEJ, Johansen-Berg H, Bannister PR, De Luca M, Drobnjak I, Flitney DE, Niazy RK, Saunders J, Vickers J, Zhang Y, De Stefano N, Brady JM, Matthews PM. Advances in functional and structural MR image analysis and implementation as FSL. Neuroimage. 2004; 23:S208-219. [PubMed: 15501092]

Smoski MJ, Felder J, Bizzell J, Green SR, Ernst M, Lynch TR, Dichter GS. fMRI of alterations in reward selection, anticipation, and feedback in major depressive disorder. Journal of Affective Disorders. 2009; 118:69-78. [PubMed: 19261334]

Woolrich MW, Ripley BD, Brady M, Smith SM. Temporal autocorrelation in univariate linear modeling of FMRI data. Neuroimage. 2001; 14:1370-1386. [PubMed: 11707093]

Zink CF, Pagnoni G, Chappelow J, Martin-Skurski M, Berns GS. Human striatal activation reflects degree of stimulus saliency. Neuroimage. 2006; 29:977-983. [PubMed: 16153860] 


\section{Incentive Trials Nonincentive Trials}
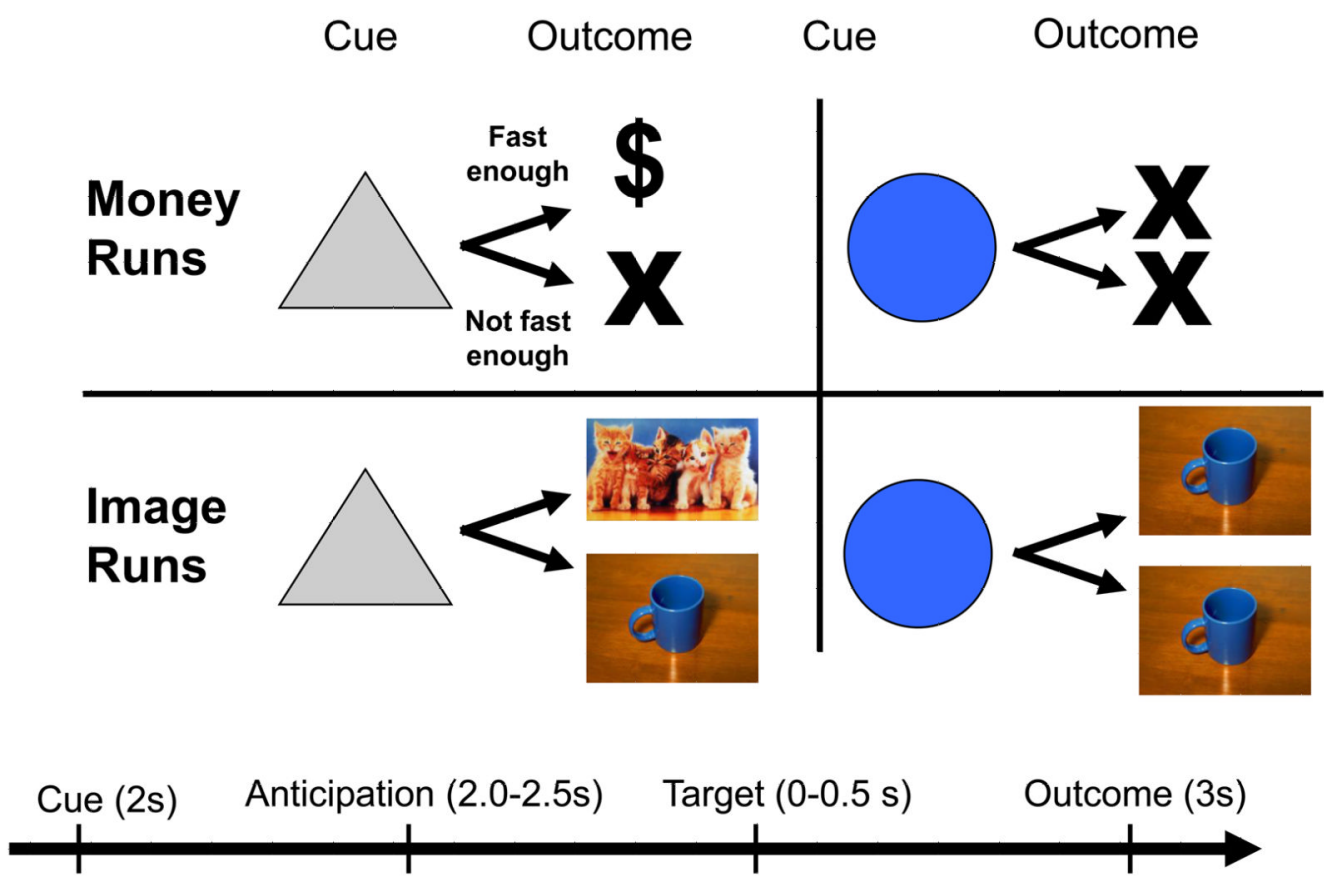

Figure 1.

Modified MID task. Participants alternated completing "money" and "image" runs, denoted by a 10 -sec instructional screen at the start of each run. Each trial consisted of a cue (i.e., a triangle indicated an incentive trial, a circle indicated a non-incentive trial), an anticipatory delay, a target, and outcome feedback. 


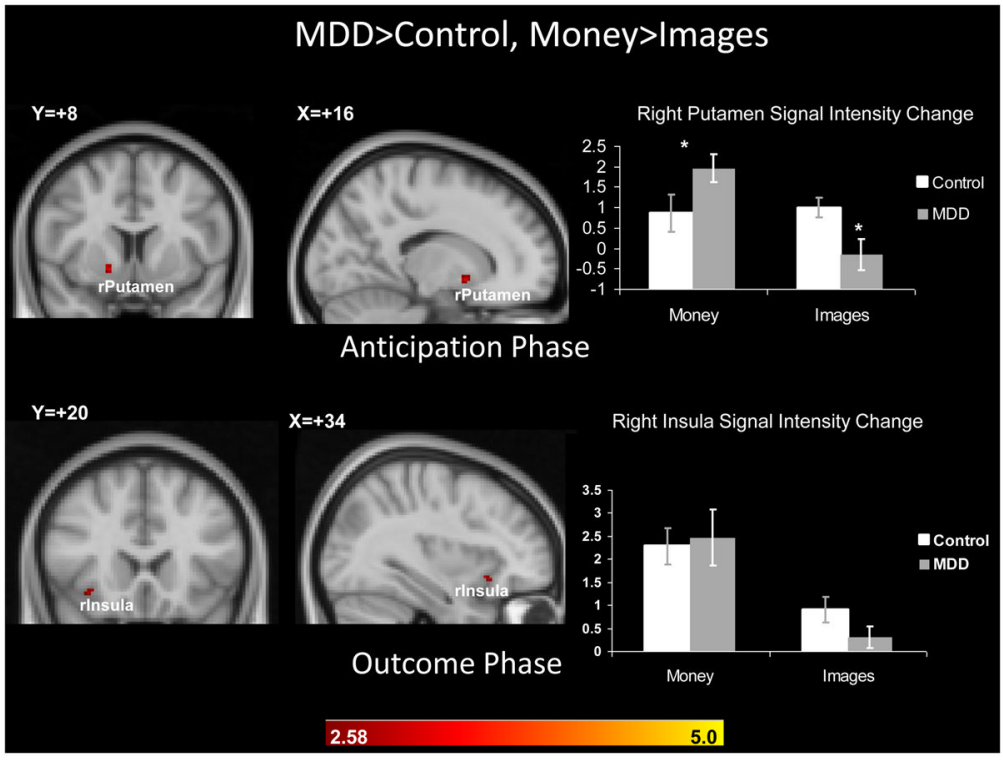

Figure 2.

Key brain areas showing significant Group $\times$ Reward Type effects during reward anticipation and outcome. Starred bars on graphs differ at $\mathrm{p}<.05$ in follow-up $t$ tests. 
Table 1

Demographic and symptom severity information for control and MDD participants. Two-tailed p-values for between-group $t$ tests or chi-squared analyses are presented in the final column.

\begin{tabular}{|l|c|c|c|}
\hline & $\begin{array}{c}\text { Depressed Subjects, } \mathbf{n}=\mathbf{9} \\
\text { Mean (SD) }\end{array}$ & $\begin{array}{c}\text { Control Subjects, } \mathbf{n}=\mathbf{1 3} \\
\text { Mean (SD) }\end{array}$ & $\boldsymbol{p}$-value \\
\hline Age & $34.4(15.1)$ & $26.2(6.3)$ & 0.10 \\
\hline Race & & & 0.51 \\
\hline African American & $11 \%$ & $15 \%$ & \\
\hline Caucasian & $67 \%$ & $77 \%$ & \\
\hline Asian & $22 \%$ & $8 \%$ & \\
\hline Hispanic Ethnicity & $11 \%$ & $0 \%$ & 0.22 \\
\hline NAART VIQ & $108.8(12.8)$ & $110.2(9.9)$ & 0.76 \\
\hline BDI & $16.7(4.9)$ & $0.8(1.5)$ & $<0.00001$ \\
\hline
\end{tabular}

NAART VIQ: North American Adult Reading Test

BDI: Beck Depression Inventory, $2^{\text {nd }}$ Edition 
o

อิ

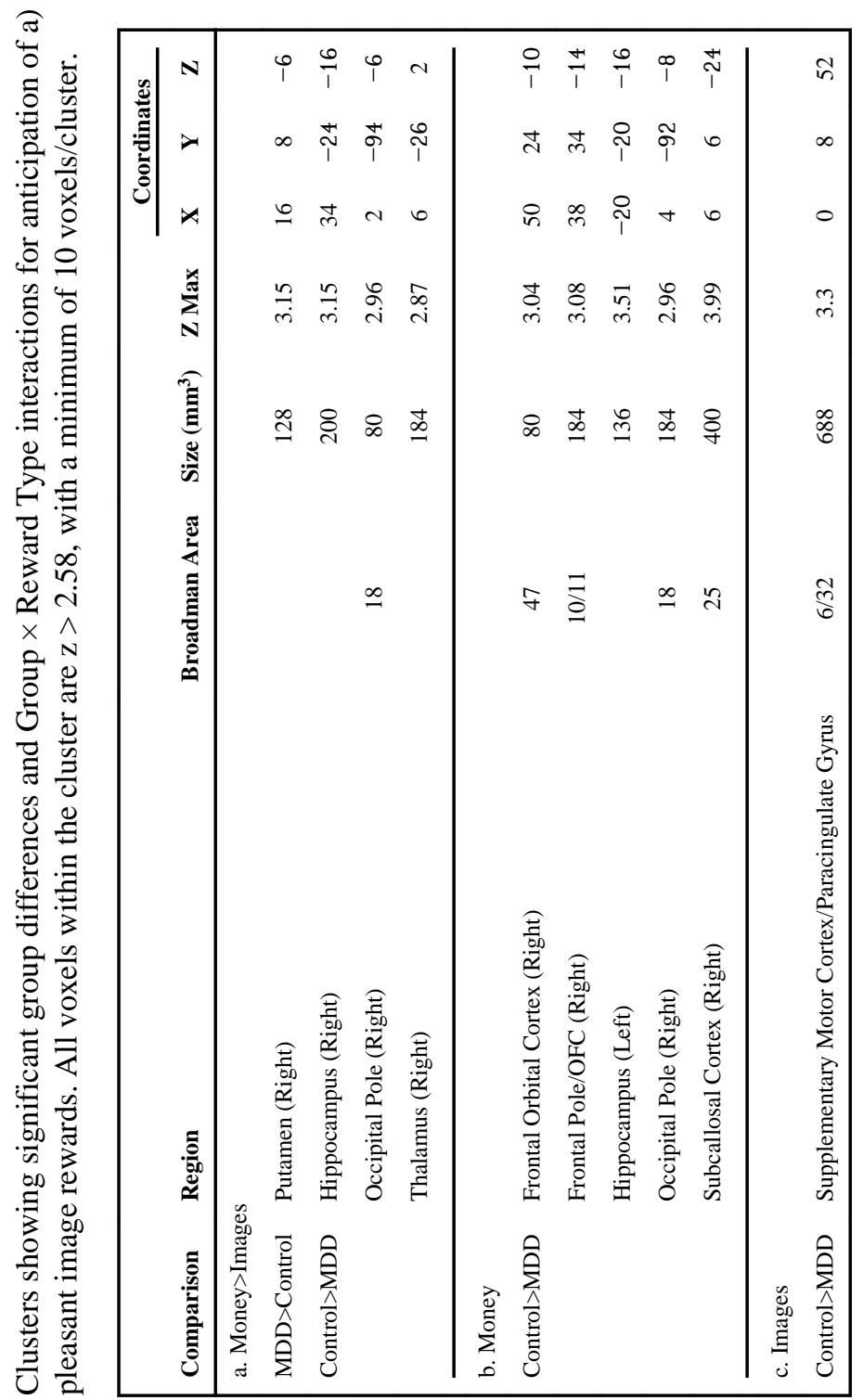




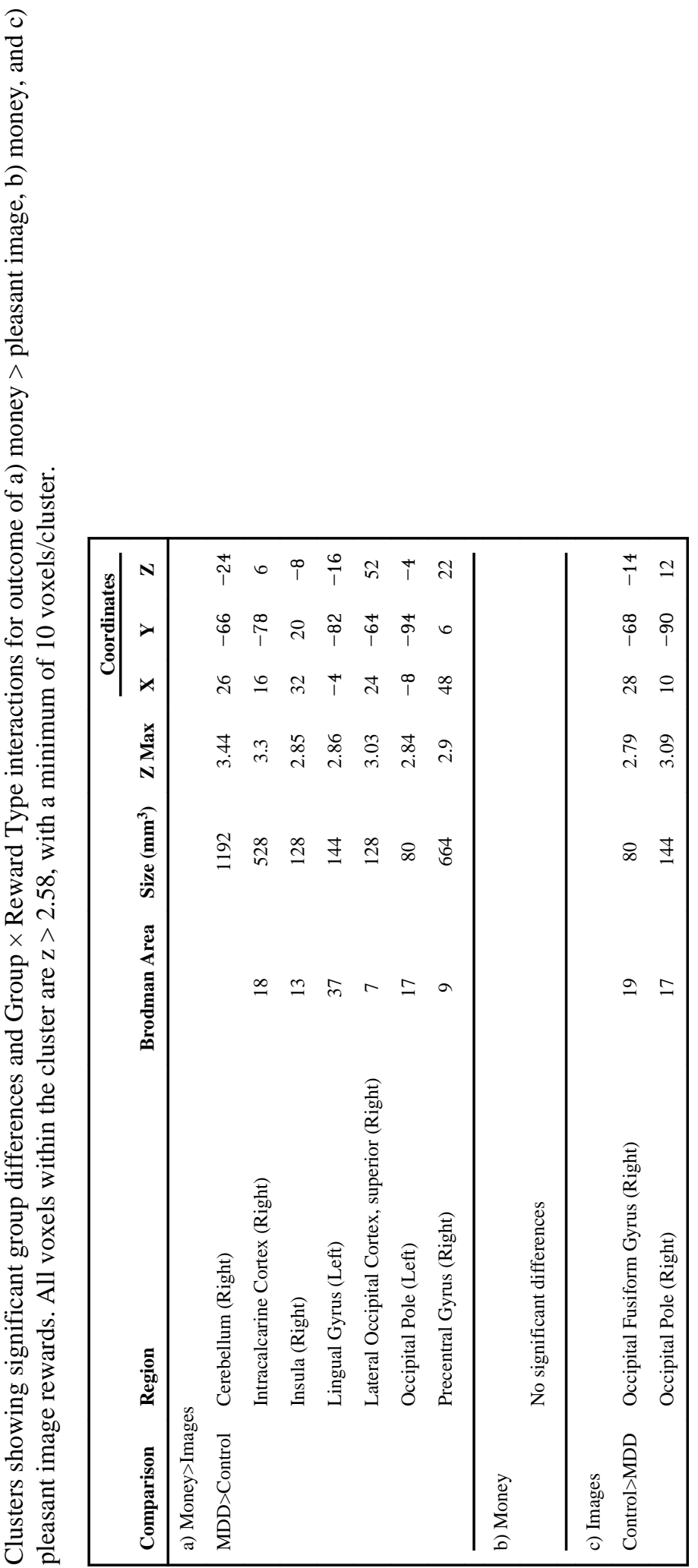

